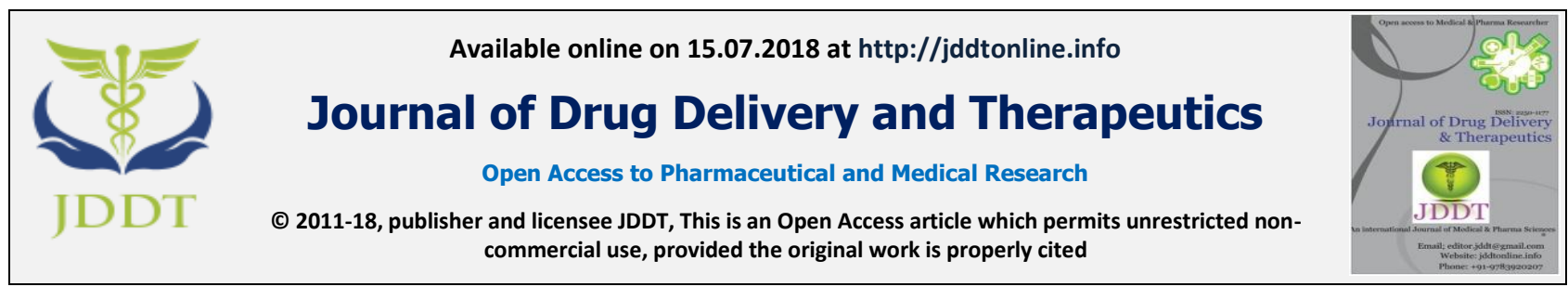

Open $\odot$ Access

Review Article

\title{
GASTRO RETENTIVE NON-FLOATING DRUG DELIVERY APPROACH: A REVIEW
}

\author{
Ramanathan $\mathbf{M}^{*}$, Manikandan S, Subramanian L, Dr. Solairaj P \\ Department of Pharmaceutics, Sankaralingam Bhuvaneswari college of Pharmacy, Sivakasi, India
}

\section{ABSTRACT}

Gastro retardation is attractive approach to enhance bioavailability of narrow absorption window drugs. Drug retardation in stomach is more beneficial for certain gastro intestinal disorders like gastro esophageal reflex disease, ulcer and other gastro intestinal conditions. Gastric site retardation is one of the challenging technique, however various techniques are applied to keep the drug and achieve the enhancement of bioavailability. To achieve gastro retentive commonly two approaches are considered, they are floating drug delivery system and non-floating gastro retentive drug delivery system. The floating drug made-up by low density polymers, this approach was not fit many of the drug molecule and floating drug has some limitations like sufficient level fluids in stomach and not to be dosed just before going to bed. In this review describe the various high density (sinking) system (non-floating) drug delivery systems like super porous hydrogels, expanding system, bio/mucoadhesives system, and magnetic system and mechanism, desirable drug characters, advantages and disadvantages.

Keyword: Bioavailability, gastro retentive, magnetic system, mucoadhesive, non-floating and sinking system.

Article Info: Received 18 Feb, 2018; Review Completed 23 May 2018; Accepted 24 May 2018; Available online 15 July 2018



Cite this article as:

Ramanathan M, Manikandan S, Subramanian L, Dr. Solairaj P, Gastro retentive non-floating drug delivery approach: a review, Journal of Drug Delivery and Therapeutics. 2018; 8(4):20-23 DOI: http://dx.doi.org/10.22270/jddt.v8i4.1725

Ramanathan. M, Department of Pharmaceutics, Sankaralingam Bhuvaneswari College of Pharmacy, Sivakasi, Tamilnadu, India

\section{INTRODUCTION}

Gastro retentive drug delivery system improves drug efficiency, reduce frequency of administration, enhance bioavailability and better patient's compliance. Several gastro retentive drug delivery approaches being designed and developed, sinking (high density) systems (that is retained in the bottom of the stomach), floating (low density) systems that causes buoyancy in gastric fluid, mucoadhesive systems that causes bio adhesion to stomach mucosa, extendible or swellable systems which exact delivery of drug dosage in stomach. ${ }^{1}$

Floating systems or hydro dynamically controlled systems that have float the tablet over the stomach contents for a prolonged period of time. ${ }^{2}$ The drug is released slowly at the desired rate from the system, after the drug release the residual system is emptied from the stomach. These results in an increased gastro retardation time (GRT) for the system buoyancy a minimal gastric content needed. Sinking high density approach involves formulation of dosage forms with the density that must exceed density of normal stomach content ${ }^{1}$. Mucoadhesive dosage forms designed prolonged retention at the gastric site, providing a controlled rate of drug release for improved therapeutic outcome. Extendible dosage (swellable) form in the stomach will withstand gastric transit if it bigger than pyloric sphincter ${ }^{1}$. The current review brief about various non-floating gastroretentive approaches of drug delivery systems. The successful development of gastric retardation drug delivery systems requires an understanding of anatomy and physiology of gastro intestinal tract (GIT) and physiochemical character of drug. 


\section{A glance of gastro intestinal tract}

The stomach is divided into three regions, fundus, body, and antrum (pylorus). The proximal part made of fundus and body acts as a reservoir for undigested material, whereas the antrum is the main site for mixing motions and act as a pump for gastric emptying by propelling actions. Gastric emptying occurs during fasting as well as fed states. The pattern of motility is differ in the two states. During the fasting state series of electrical events take place, which cycle both through stomach and intestine every 2 to 3 hours, this is called interdigestive myloelectric cycle or migrating myloelectric cycle (MMC), which is further divided into following 4 phase. ${ }^{3,4}$

1. Phase I (basal phase) lasts from 30 to 60 minutes with rare contractions. ${ }^{5}$

2. Phase II (preburst phase) lasts for 20 to 40 minutes with intermittent action potential and contractions. As the phase progresses the intensity and frequency also increases gradually. ${ }^{6}$

3. Phase III (burst phase) lasts for 10 to 20 minutes. ${ }^{7}$ It includes intense and regular contractions for short period. It is due to this wave that all the undigested material is swept out of the stomach down to the small intestine. It is also known as the housekeeper wave.

4. Phase IV lasts for 0-5 minutes and occurs between phases III and I of 2 consecutive cycles. ${ }^{8}$

After consumption of meal, the pattern of contractions changes from fasted to feed state. This is known as digestive motility pattern and comprises continuous contractions as in phase II of fasted state. These contractions result in reducing the size of food particles (to less than $1 \mathrm{~mm}$ ), which are propelled toward the pylorus in a suspension form. During the fed state onset of migrating myloelectric cycle is delayed resulting in slowdown of gastric emptying rate. $6,9,10$

\section{Gastro retentive drug delivery system desirable} characters

1. Drugs that act locally in the stomach e.g., Antacids and Misoprostol ${ }^{\mathbf{1 , 1 1}}$

2. Drugs that are primarily absorbed in the stomach. e.g. Calcium supplements, Chlordiazepoxide and Cinnarazine.

3. Drugs those are poorly soluble at an alkaline $\mathrm{pH}$.

4. Drugs that have a narrow window of absorption. e.g., Riboflavin and Levodopa.

5. If the drugs disturb normal colonic microbes.

6. Drugs that is unstable in the intestinal or colonic environment. e.g. Ranitidine and Metronidazole.

7. Drugs with variable bioavailability. E.g. Sotalol $\mathrm{HCl}$

\section{Advantages of Gastro retentive drug delivery} system $^{6,10}$

1. Enhanced drug absorption.

2. Controlled drug delivery.

3. Delivery of drugs for local action in the stomach.

4. Minimizing the mucosal irritation due to slow and controlled rate.

5. Highly suitable for the treatment of gastrointestinal disorders such as gastro-esophageal reflux.

6. Simple and conventional equipment is enough for formulate.

7. Site-specific drug delivery.

Disadvantages of gastroretentive drug delivery systems $^{6,11}$

1. Unsuitable for drugs with limited acid solubility.

2. Unsuitable for drugs that are unstable in acidic environment.

3. Drugs that irritates or causes gastric lesions on slow release. E.g. Aspirin \& NSAID's

4. Unsuitable drugs that absorb selectively in colon.

5. Floating drug delivery systems require high fluid level in stomach to float and work effectively.

\section{Classification of GRDDS}

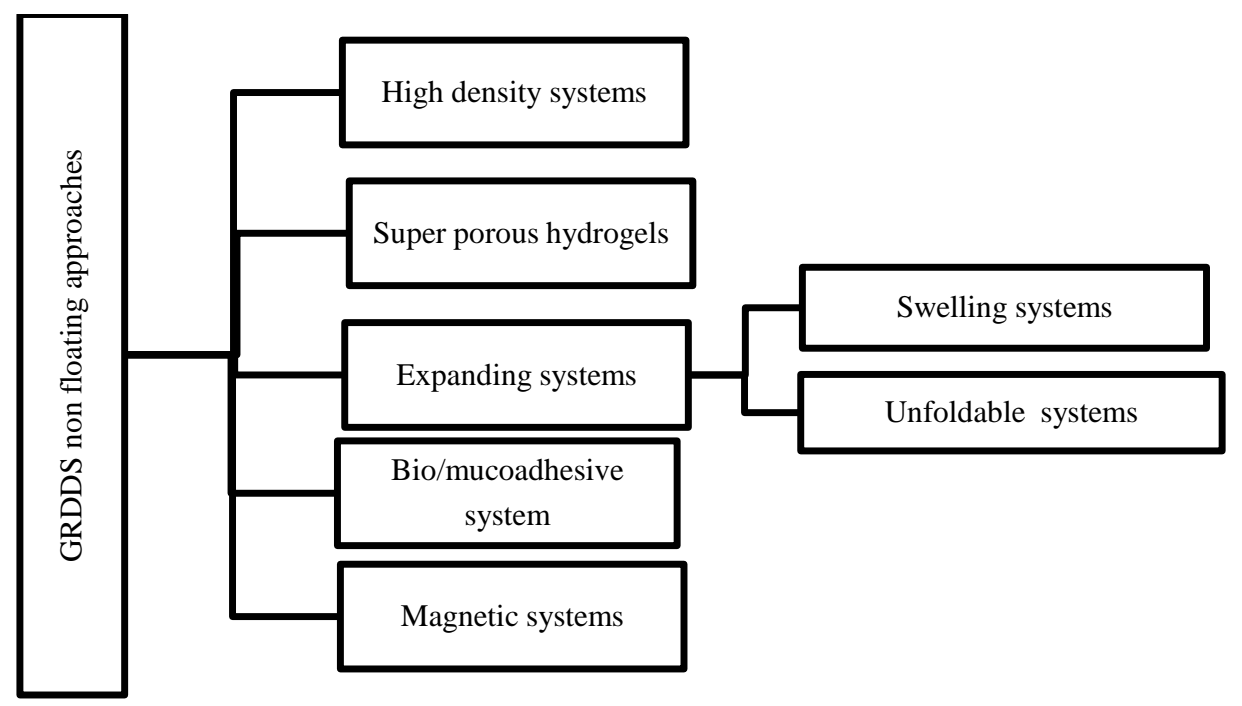




\section{Sinking (High Density) Systems}

These systems, which have a density of $3 \mathrm{~g} / \mathrm{cm}^{3}$ are retained in the stomach and capable of withstanding its peristaltic movements. The only drawback with these systems is that, it is technically difficult to manufacture them with a large amount of drug (>50\%) and achieve required density of $2.4-2.8 \mathrm{~g} / \mathrm{cm}^{3}$. Diluents such as barium sulphate $($ density $=4.9)$, zinc oxide, titanium oxide, and iron powder must be used to manufacture such high-density formulation. 5,6

\section{Super porous hydrogel systems}

The approach to improve gastric retention time (GRT) by super porous hydrogels of average pore size $>100$ micro meter, swell to equilibrium size within a minute due to rapid water uptake by capillary and wetting through numerous interconnected open pores.

They swell to a large size (swelling ratio: 100 or more) and are intended to have sufficient mechanical strength to withstand pressure by gastric contraction. This is advised by co-formulation of hydrophilic particulate material. $^{1,6}$ This is achieved by co-formulation of a hydrophilic particulate material, and Ac-Di-Sol (crosscarmellose).

\section{Swelling system (Expandable systems)}

The swelling is usually results from osmotic absorption of water. The dosage form in the stomach will withstand gastric transit if it bigger than pyloric sphincter. These system may be reffered as a 'plug type system' the device gradually decreases in volume and rigidity. The final small form of dosage enabling evacuation following drug release from the system. ${ }^{4,11}$

\section{Unfoldable and swellable systems}

Unfolding systems are systems which are actually of larger size but they are folded to decrease size and kept in capsules. In stomach these systems comes out of capsules and unfolds to larger size. The important factor for unfolding system is shape memory. They should have sufficient shape memory such that they retain their unfolded (expanded) shape in stomach against gastric motility and not get folded again and escape out till the desired time interval.

Unfoldable systems are available in various shapes. Caldwell et al, proposed different geometric forms like tetrahedron, ring or planar membrane, 4-lobed, disc and 4-limbed cross form of bioerodible polymer compressed within a capsule. Expandable systems have some drawbacks like easily hydrolysable, biodegradable polymers relatively short-lived mechanical shape, difficult to industrialize and not cost effective., ${ }^{42,14}$

\section{Muco / Bio-adhesive systems}

Muco/Bioadhesive drug delivery systems (BDDS) are used to make delivery of drug in lumen of GIT and facilitate drug absorption in a site specific site. This approach involves the use of bioadhesive polymers, which can adhere to the epithelial surface in the stomach. The basis of mucoadhesion is that a dosage form can stick to the mucosal surface by different mechanisms. The drawback of Gastric mucoadhesion does not tend to be strong enough to impart to dosage forms the ability to resist the strong propulsion forces of the stomach wall. The commonly used excipients are polycarbophil, carbopol, lectins, chitosan and gliadin, etc. ${ }^{1,4,12}$. As the mucoadhesivity improves the retention time of the formulation at the site of absorption in GIT, so it helps a sustained release effect for a longer period ${ }^{13}$

\section{Magnetic Systems}

Based upon the principle that dosage form contains a small internal magnet, and a magnet placed on the abdomen over the position of stomach can enhance the gastro retardation time. Although magnetic system seems to work, the external magnet must be positioned with a degree of precision that might compromise patient compliance. ${ }^{12}$

\section{DISCUSSION}

Novelty in gastro retardation is attractive and most beneficial technique for narrow absorption window drugs. Retarding the drug in gastric site is real challenging work, and to face lot of biological constrains, formulation and patients side considerations. These techniques are very much fit for ulcer curative antibiotic therapies, those drugs are used in high concentration in conditions like helicobacter pylori infection eradication. Prolonged drug release can produce better bioavailability and good patient compliance. However the complexity of pharmacokinetic and pharmacodynamics parameters invivo studies are required to establishment of specific drug dosage. All types of gastroretardation techniques are have its own merits and demerits. Nowadays lot of research in this field has provided variety of techniques to adopt and make designer drug in gastro retardation.

\section{ACKNOWLEDGEMENT}

The authors are thankful to the management and Principal of Sankaralingam Bhuvaneswari College of Pharmacy, Sivakasi, for providing laboratory, library, internet facilities and constant encouragement. 


\section{REFERENCES}

1. Nayak AK, Maji R, Das B, Gastroretentive drug delivery system: a review, Asian journal of pharmaceutical and clinical research, 2010; 3(1).

2. Paresh Mohan et al., A review on natural polymers approaches to floating drug delivery system, Asian journal of pharmaceutical research and development, Sep-Oct. 2013; 145-159.

3. Choure $\mathrm{S}$ et al., formulation and evalution of floating tablets of nizatidine, International journal of research Ayurveda pharm, Mar-Apr 2015; 6(2)

4. Suryawanshi A, Hiremath S.P. Floating drug delivery system a review, American journal of pharmtech research, 2012; 2(1).

5. Bhardwaj V, Nirmala, Harikumar S.L. Floating drug delivery system a review, Pharmacophore, 2013; 4(1):26-38.

6. Badoni A et al., Review on gastro retentive drug delivery system, The pharma innovation,2012; 1(8)

7. Arunachalam A et al., Floating drug delivery system:A review, Int. J. Res. Pharm. Sci., 2011; 2(1):76-83.

8. Chaudhary P, Nirmala, Harikumar S.L. Approaches for gastroretentive drug delivery system-a review, Journal of drug delivery and therapeutics, 2014; 4(3):14-24. doi:10.22270/jddt.v4i3.834
9. Saravanan D et al., Formulation and evaluation of ofloxacin floating tablets using HPMC, International journal of pharmacy and pharmaceutical science, 2011;3(1)

10. Bhalla $\mathrm{N}$ et al., An overview on various approaches to oral controlled drug delivery system via gastroretentive drug delivery system, International research journal of pharmacy, 2012; 3(4)

11. Sarojini.S and Manavalan R, An overview on various approaches to gastro retentive dosage forms, international journal of drug development and research, Jan-March, 2012; 4(1):01-13.

12. Kadam Shashikant $\mathbf{M}$ et al., Review on floating drug delivery system: an approach to oral controlled deug delivery via gastric retension, international journal of research in Ayurveda and pharmacy, 2011; 2(6):1752-1755.

13. Abdeltwab et al., Novel gastro retenntive polymeric microsphers: an approach for increased bioavailability and A once daily dosing of terbutaline sulphate, International journal of pharmacy and pharmaceutical science, 2016;8(8).

14. Narang et al., An updated review on: Floating drug delivery system, International journal of applied pharmaceutics, 2011; $3(1)$. 